\title{
Arbeitsstress
}

\section{Eine neue Berufskrankheit?}

\begin{abstract}
Die moderne Arbeitswelt erleben viele Menschen als psychische Belastung. Andererseits sind über $80 \%$ aller Erwerbstätigen der Auffassung, dass ihre Arbeit sie fit hält. Wie lassen sich diese Widersprüche erklären? Gibt es gesicherte Anhaltspunkte dafür, dass Arbeitsstress zu Organschädigungen führen kann, und wie sieht es mit der Anerkennung als Berufskrankheit aus?
\end{abstract}

— Nach einer neueren Befragung ist jeder vierte Beschäftigte der Ansicht, dass arbeitsbedingter Stress gesundheitsschädigend sei. „Ein Viertel der Ausfallzeiten durch eine arbeitsbedingte Erkrankung geht auf das Konto von arbeitsbedingten Stress", sagte Prof. Klaus Scheuch, Dresden. Die Arbeitsunfähigkeit aufgrund psychischer Erkrankungen ist in den letzten zehn Jahren um ca. 70\% gestiegen und auch bei der Erwerbsminderung spielen psychische Erkrankungen die wichtigste Rolle.

\section{Zunehmende Überforderung}

Die moderne Arbeitswelt fordert höhere Qualifikationen, mehr Flexibilität, größeren Einsatz und mehr Verantwortung als früher. „Die zunehmende Unsicherheit und der ständige Druck, Neues zu lernen, hat zur Folge, dass sich Arbeit und Freizeit zunehmend vermischen", so Scheuch. Immer mehr Beschäftigte haben das Gefühl, den Anforderungen nicht mehr gerecht werden zu können.

Die dauerhafte Belastung kann zu psychischen und körperlichen Beschwerden führen. Initial klagen Betroffene über Erschöpfung und herabgesetzte Vigilanz. Hinzu kommen Leistungsminderung und Motivationsverlust.

\section{Vom Tinnitus bis zum Diabetes}

Stress selbst ist keine Krankheit, sondern ein Anpassungsprozess, der als verursachender oder begünstigender Faktor in Krankheiten münden kann. Andererseits kann Stress auch zur persönlichen Weiterentwicklung beitragen und Widerstandskraft und Leistungsfähigkeit erhöhen - allerdings nur dann, wenn die Probleme zu bewältigen sind. „Da Stress etwas Unspezifisches ist, gibt es keine spezifischen Stresskrankheiten, sondern nur mögliche Auswirkungen auf unterschiedlichste Organ- und Funktionssysteme“, so Scheuch.

Das Spektrum der Erkrankungen, bei denen psychosomatische Faktoren eine Rolle spielen, reicht von der KHK über die Hypertonie und Herzrhythmusstörungen bis zu Magengeschwüren und Migräne. Auch Diabetes mellitus, chronisch entzündliche Darmerkrankungen, Allergien, vermehrte Infektionsbereitschaft, Migräne, Tinnitus, Menstruationsstörungen, Depressionen, Angstzustände und Hauterkrankungen können durch Stress ungünstig beeinflusst werden. Warum bei dem einen Patienten das Herz oder der Blutdruck, beim anderen der Magendarmtrakt und beim Dritten die Psyche betroffen ist, kann man allerdings bisher nicht erklären.

\section{Nicht als Berufskrankheit anerkannt} Kann oder sollte Stress als Berufskrankheit anerkannt werden? „Nur in Einzelfällen kann ein Arbeitsunfall bei psychischen Fehlbelastungen wie z.B. einem traumatischen Ereignis anerkannt werden“, so Scheuch. Nach geltendem Recht sei eine Berufskrankheit "Stress" kaum möglich, da Stress ein komplexes Geschehen ist, in dem die versicherungsrechtlichen Kausalitätskriterien schwierig bis gar nicht nachzuweisen sind. Auch ist es kaum möglich, bestimmte Personengruppen als besonders stressgefährdet zu identifizieren, da Stress in allen Berufen auftreten kann.

\section{Stress ist subjektiv}

Das Phänomen Stress entsteht jedoch nicht nur bei Über- sondern auch bei Unterforderung. Deshalb dürften Ar-

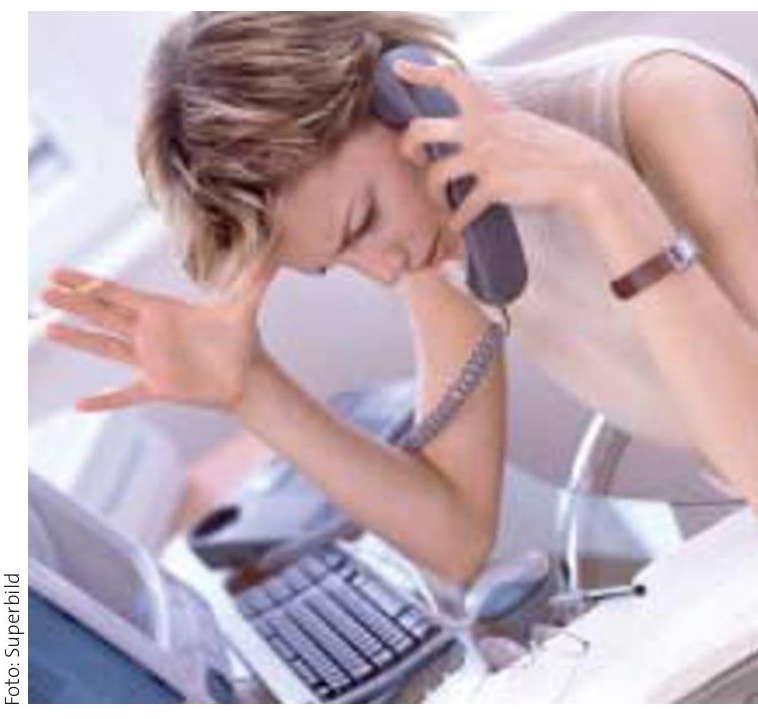

Ist sie der Belastung gewachsen?

beitslose stärker stressgefährdet sein als Beschäftigte. Die entscheidende Rolle spielt nicht die objektive Belastung wie z. B. bei Gefahrenstoffen, sondern die individuelle Prägung, wobei Persönlichkeitsmerkmale, Verhaltens- und Bewältigungsstile, Bedürfnis- und Motivstruktur für das individuell unterschiedliche Stressempfinden entscheidend sind. Hinzu kommt, dass auch außerberufliche Einflüsse bei der Bewältigung des Arbeitsstresses eine Rolle spielen.

\section{Bessere Arbeitsbedingungen}

Die Bedeutung arbeitsbezogener psychischer Fehlbelastungen wird in den nächsten Jahren zunehmen. Hier ergeben sich neue Aufgaben der Prävention und Gesundsheitsförderung. Vorrangiges Ziel muss es sein, die psychische Belastung am Arbeitsplatz zu minimieren. Dazu gehören nach Scheuchs Ansicht ganzheitliche Anforderungen, eine Vielfalt der Tätigkeit, Möglichkeiten der sozialen Interaktion, Autonomie, Lern- und Entwicklungsmöglichkeiten, Sinnhaftigkeit und Belohungsmöglichkeiten. Allerdings kann auch eine "gute“ Arbeit zur Selbstausbeutung mit Vernachlässigung psychischer und sozialer Regenrationsprozesse führen mit der Folge stressbedingter Beschwerden.

\section{Dr. med. Peter Stiefelhagen}

- Quelle: Internistenkongress 2008 in Wiesbaden 